\title{
Pulse coding linearization for Brillouin optical time-domain analysis sensors
}

\author{
Jon Mariñelarena ${ }^{1}$, Haritz Iribas ${ }^{1}$, And Alayn LoAyssa ${ }^{1}$, $^{*}$ \\ ${ }^{1}$ Institute for Smart Cities, Departamento Ingeniería Eléctrica y Electrónica, Universidad Pública de Navarra, Campus de Arrosadia s/n, 31006 Pamplona, Spain \\ *Corresponding author: alayn.loayssa@unavarra.es \\ Compiled October 17, 2018
}

We introduce a simple method to extend the performance of pulse coding techniques in their application to Brillouin optical time-domain analysis sensors (BOTDA). It is based on using a simple logarithmic processing on the detected probe wave that compensates the deviation from linearity of the sensor response for long code lengths. The technique ensures that the accumulated effect of a sequence of pulses is equal to the linear addition of the effects of the individual components, which is the essential condition to ensure a correct decoding of the probe gain measurement. We experimentally demonstrate compensation of the Brillouin frequency shift error induced by the accumulated gain nonlinearity. Furthermore, a proof-ofconcept $80-\mathrm{km}$ sensing link within a total $200-\mathrm{km}$ fiber loop demonstrated better than 2-MHz precision with 2m spatial resolution.

(C) 2018 Optical Society of America

OCIS codes: 060.2370 Fiber optics sensors, 290.5900 Scattering, stimulated Brillouin

http://dx.doi.org/10.1364/ao.XX.XXXXXX

Brillouin optical time-domain analysis (BOTDA) sensors have been extensively researched in the last years due to the long range measurements of temperature and strain that they can provide. These measurements are very valuable to monitor the integrity of large structures such as oil and gas pipelines, electric high-voltage cables or railways. However, the range of BOTDA sensors is ultimately constrained by the attenuation of the optical fiber used for sensing because the maximum pump and probe powers that can be deployed are limited by the onset of nonlinear and nonlocal effects. Multiple solutions have been proposed to increase the range of BOTDA sensors, but one of the most powerful is the deployment of pump pulse coding [1]. These methods are based on launching in the fiber coded sequences of pulses and processing the detected probe signals so as to obtain, after a decoding process, a probe intensity response equivalent to that of the single-pulse BOTDA, but with an enhancement in signal-to-noise ratio (SNR), known as coding gain, that increases with the code length. This enhancement is due to the increased pump energy in the fiber and it does not compromise the spatial resolution of the sensor.

Despite its potential, the application of pulse coding methods so far has been constrained by the linearity assumption, i.e., the assumption that the effects of interaction with multiple pulses in a coded sequence on the probe accumulates linearly. This is required for the decoding process to work properly. However, this limits the maximum achievable coding gain because for long codewords the accumulated gain provided by the pulse sequence for optical frequencies close to the Brillouin peak becomes large, so that the linearity approximation is no longer satisfied. Several solutions have been proposed to mitigate this constrain. One alternative is the use of color coding, which is based on introducing an additional optical frequency hopping to the pulse sequence [2]. This additional and continuous frequency change of the pump pulses of a given sequence reduces the total gain experienced by the probe because only part of the pump pulses are close to the Brillouin gain peak. Another proposal is the use of bipolar coding, which is based on using codes that include pulses that are upshifted or downshifted in optical frequency from the probe so that they induce either gain or loss on the probe wavefront [3]. This ensures that the total gain or loss experienced by the probe after transversing the fiber is small; hence, linear conditions prevail. This approach has demonstrated very good performance at the cost of a rather increased setup complexity.

In this work, we introduce a much simpler approach to attain pulse coding linearity by a very simple processing of the detected probe wave. This new technique entails linearizing the sensor response so that, no matter the amount of gain experienced by the probe, the accumulated effect of a sequence of pulses is equal to the linear addition of the effects of the individual components. The method is experimentally demonstrated in a 200-km fiber link obtaining a remarkable performance.

In pulse coding there is a fundamental requirement for linearity in order to obtain proper results in the decoding process. In a single-pulse BOTDA sensor, the objective is to determine the Brillouin gain experienced by the probe wave at every position in the fiber, $g(z)$. In practical deployments, the variation of the detected CW probe intensity, $\Delta I_{C W}$, due to interaction with the 
pump pulse at $z$ is used as a proxy for $g(z)$ :

$$
\begin{aligned}
\Delta I_{C W}(t) & =I_{C W}(t)-I_{C W_{L}} \exp (-\alpha L) \\
& =I_{C W_{L}} \exp (-\alpha L)[\exp (g(z)-1] \\
& \approx I_{C W_{L}} \exp (-\alpha L) g(z)
\end{aligned}
$$

where $I_{C W}(t)$ is the detected intensity of the probe wave exiting the fiber at $t=(L-z) / v_{g}$, with $v_{g}$ the group velocity of the optical waves and $I_{C W_{L}}$ is the probe power injected in the fiber of length $L$ and attenuation coefficient $\alpha$. In this expression, the approximation of the last right-hand side term assumes that the gain is small. In pulse-coded BOTDA, the methods applied so far have also relied on the measurement of the total intensity variation experienced by the probe when interacting with particular codeword sequences of pulses in the fiber, $\Delta I_{C W_{T}}$ :

$$
\begin{aligned}
\Delta I_{C W_{T}}(t) & =I_{C W_{L}} \exp (-\alpha L)\left[\exp \left(\sum_{i=1}^{L_{C}} g_{i}\left(z_{i}\right)\right)-1\right] \\
& \approx I_{C W_{L}} \exp (-\alpha L) \sum_{i=1}^{L_{C}} g_{i}\left(z_{i}\right)
\end{aligned}
$$

where $g_{i}$ is the gain experienced by the probe due to its interaction with the $i$-th pulse in the codeword sequence at a location $z_{i}$ along the fiber, and $t$ refers again to the probe wavefront exiting the fiber prior to detection. Notice that the different $g_{i}$ are identical to $g\left(z_{i}\right)$ for the single-pulse case, a fact that is used in the decoding process. Again, the last approximation on the right-hand side of Eq. (2) is just valid when $\sum_{i=1}^{L_{C}} g_{i}\left(z_{i}\right)$ is small. However, contrary to the single-pulse case, this assumption becomes unreasonable as the code length, and hence the total gain, increases. This makes the recovery of $g(z)$ from the measured $\Delta I_{C W_{T}}$ for each codeword to fail, because the decoding process intrinsically requires that the effects of multiple pulses accumulate linearly. Altogether, this linearity requirement constrains the maximum code length that can be deployed in BOTDA sensors and, with it, the maximum achievable SNR enhancement and overall measurement range.

We propose an alternative pulse coding implementation method that is based on using a linearized version of the probe intensity variation. This is obtained by calculating the natural logarithm of the detected probe intensity wave, $I_{C W_{T}}$, and subtracting its DC component. The linearized variation of the probe intensity due to its interaction with a given codeword is then given by:

$$
\Delta I_{C W_{T}}^{\prime}(t) \equiv \ln \left(I_{C W_{T}}(t)\right)-\ln \left(I_{C W_{L}} \exp (-\alpha L)\right)
$$

From this definition, it is easy to demonstrate that working with $\Delta I_{C W_{T}}^{\prime}$ instead of $\Delta I_{C W_{T}}$ provides linearity to the coded signal no matter what the total gain experienced by the probe is:

$$
\begin{aligned}
\Delta I_{C W_{T}}^{\prime}(t)= & \ln \left(I_{C W_{L}} \exp (-\alpha L) \exp \left(\sum_{i=1}^{L_{C}} g_{i}\left(z_{i}\right)\right)\right) \\
& \quad-\ln \left(I_{C W_{L}} \exp (-\alpha L)\right) \\
= & \sum_{i=1}^{L_{C}} g_{i}\left(z_{i}\right)=\sum_{i=1}^{L_{C}} \Delta I_{C W_{i}}^{\prime}(t)
\end{aligned}
$$

where $\Delta I_{C W_{i}}^{\prime}(t)$ is the linearized probe intensity variation due to interaction of the probe wave with the $i$-th pulse.

Apart from this really simple modification, the measurement process then proceeds identically to established procedures. First, $I_{C W_{T}}(t)$ is detected for the different codewords and $\Delta I_{C W_{T}}^{\prime}(t)$ calculated for each of them, according to Eq. (2). Then, decoding is performed. For instance, for simplex or cyclic simplex coding, this involves application of the inverse code matrix.

In addition to amplification nonlinearity, the other main constrain faced by pulse coding in BOTDA is nonlocal effects due to pump pulses depletion. These effects are exacerbated in BOTDA sensors deploying coding because successive pulses in a sequence interact with probe wavefronts that have been amplified by previous pulses in the sequence [4]. This increases the energy transfer from the pump pulses to the probe wave when providing gain and hence increase pump depletion, which leads to decoding errors because the relative amplitudes of the pulses in a sequence are altered along the fiber. This constrain is also addressed in this work by deploying a method to reduce pump depletion that is based on introducing a wavelength modulation or dithering to the probe wave [4].

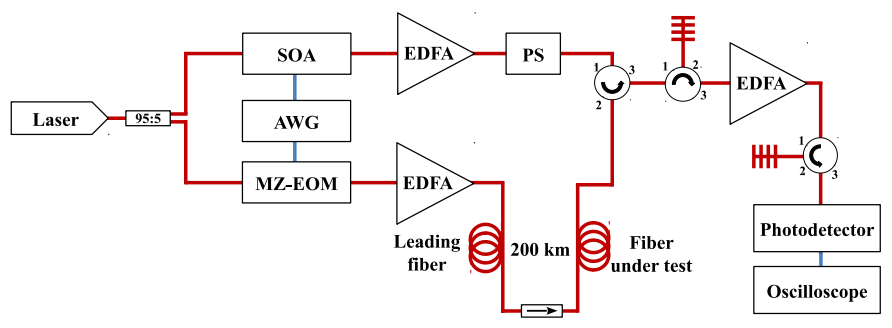

Fig. 1. Experimental setup for the BOTDA sensor with coding linearization.

In order to evaluate the performance of the proposed coding linearization method, we deploy the setup outlined in Fig. 1. This is a conventional dual-probe BOTDA setup that also incorporates the probe dithering method to mitigate first and second order nonlocal effects [4]. The optical source was a 1550-nm DFB laser, whose output was divided by a coupler into two branches. In the upper branch, a semiconductor optical amplifier (SOA) optical switch, with an extinction ratio around $50 \mathrm{~dB}$, was used to shape the pump pulses. This SOA was driven by an arbitrary waveform generator (AWG) that provided the coded sequence of pulses. Then, an erbium-doped fiber amplifier (EDFA) followed by a polarization scrambler was used to amplify the pulses to $20 \mathrm{dBm}$, which corresponds to the modulation instability threshold, and to randomize their polarization, respectively, prior to injection into the fiber under test (FUT). The pulses had 20-ns duration, which leads to a spatial resolution of $2 \mathrm{~m}$ for the sensor measurements.

A total of $200 \mathrm{~km}$ of fiber in a loop configuration were deployed, with the first half acting as leading fiber and the second as sensing fiber. Two hotspots are induced at the start and end of the sensing fiber by introducing 80-m length of fiber at both locations, in temperature-controlled thermal baths.

In the lower branch, a dual-probe wave was generated using a Mach-Zehnder electro-optic modulator (MZ-EOM) that was driven by another output of the AWG. In order to implement the modulation of the probe wave frequency or dithering, the AWG generated a microwave tone whose instantaneous frequency was modulated following a sawtooth shape with a peak-to-peak frequency deviation of $160 \mathrm{MHz}$ and a period of $4 \mu \mathrm{s}$. This frequency modulation (FM) has been shown to have two main effects [5]. First, it increases the effective Brillouin threshold of the FUT, which allows to inject a total probe wave power (per sideband) of $13 \mathrm{dBm}$, much larger than the $7 \mathrm{dBm}$ of Brillouin 
threshold measured without dithering. The second effect is to reduce first and second order nonlocal effects because the Brillouin interaction induced by the dual probe wave upon the pulse is spread in a larger frequency range [5].

An optimized cyclic code was deployed in this proof-ofconcept BOTDA sensor setup that was based on a circulant matrix of dimension $L_{c}$, with $L_{c}$ a prime number $[2,6]$. The code is generated following the recurrence expression $u_{n+1}=\left(u_{n}+n\right) \bmod L_{c}$, with $u_{1}=0$ and $\bmod$ the modulo operation. This gives the positions of pulses ("1" bits) in the first line of the code matrix (first codeword) as $p_{n}=u_{n}+1$. The rest of the lines of the matrix are obtained by simple shifting operations. Instead, the first sequence is simply repeated in a continuous loop, providing fast averaging [6]. In our implementation, the temporal distance between two of the contiguous bits of the generated cyclic code has to be equal to the period of the FM of the probe waves in order for the decoding process to work properly [4]. This ensures that, at a given location in the fiber, successive pulses in a sequence meet the probe wave with the same frequency detuning.

Finally, the probe wave was detected in an EDFA-preamplified receiver that include a couple of narrow-band fiber Bragg gratings (FBG) that were used to filter out one of the probe sidebands and the Rayleigh backscattering originated from the pump wave.

The experiments started by measuring the amplitude of the pump pulses injected in the fiber after their amplification in the EDFA. This gives information regarding the amplitude distortion introduced by the EDFA transient response, which is crucial to derive amplitude weighting values to be used in the code matrix so as to ensure a correct decoding. The maximum variation in amplitude of the pulses in the cyclic sequence was found to be around $2.5 \%$ when $L_{c}=251$ was deployed. Then, the depletion induced by the probe wave on the pump pulse sequences after traveling across the fiber was also measured. Fig. 2 highlights the general trends that the depletion increases along the pulse sequence and that shorter sequences lead to smaller depletion, as it had been observed in previous works [4]. Nevertheless, deployment of the probe dithering method makes depletion negligible from a practical point of view.

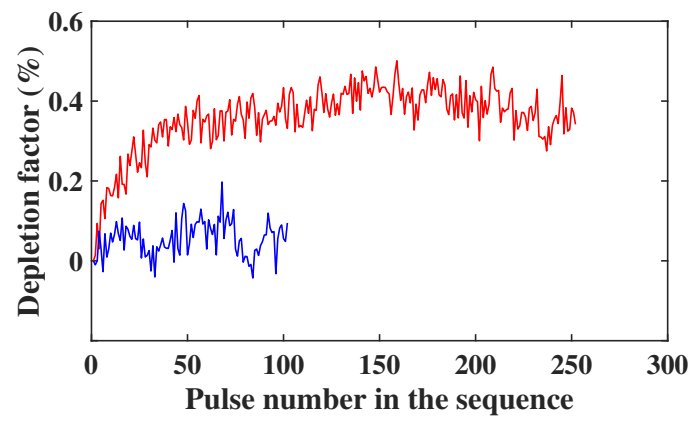

Fig. 2. Depletion of the different pulses in a code sequence for code lengths of $L_{c}=101$ (blue) and $L_{c}=251$ (red).

After characterization of the pulses, full BOTDA measurements were performed on the fiber link. Fig. 3 (a) depicts the decoded probe power variations measured along the fiber for a frequency difference between pump and probe close to the Brillouin peak gain and for different coding lengths. Notice that, as the code length is increased, the decoded signal displays increased amplitude error compared to that of a single-pulse
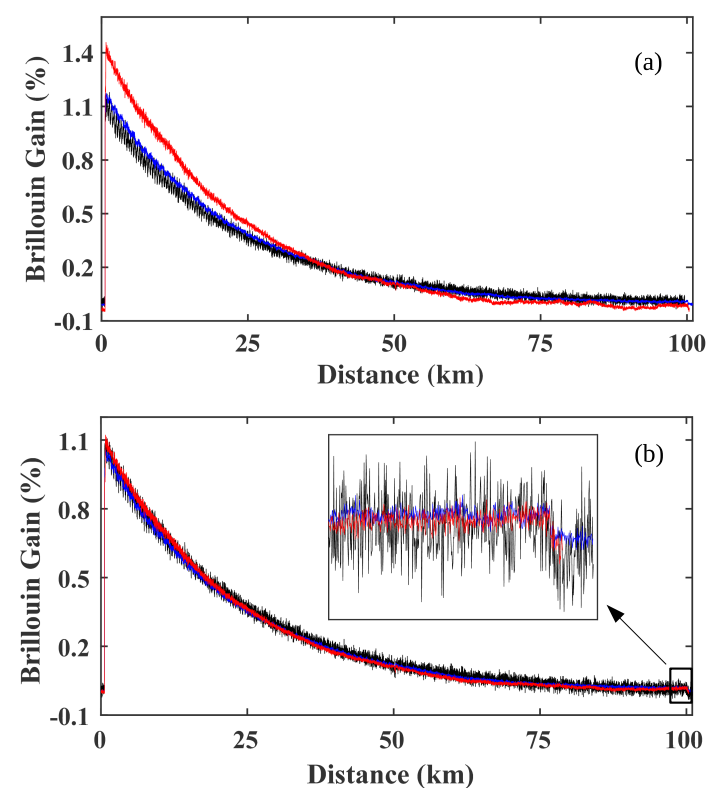

Fig. 3. Probe amplitude along the fiber (a) without and (b) with linearization of the sensor response for code lengths of $L_{c}=1$ (no coding) (black), $L_{c}=101$ (blue) and $L_{c}=251$ (red).

BOTDA. This error is due to the increased gain experienced by the probe due to its interactions with successive pulses, particularly more visible at locations close to the pulses entry in the fiber, which is where they have larger power, however, as it will be shown, errors also appear after a certain distance of the FUT. On the contrary, Fig. 3 (b) highlights that when our simple processing technique is deployed to linearize the response, the decoded probe power variation for increased code length becomes equivalent to that with no coding and the linearity error is eliminated altogether. Moreover, Fig. 4 (a) displays the spectrum measured in the hotspot at the start of the sensing fiber. This figure highlights that the deviation of linearity when deploying coding leads to a Brillouin frequency shift (BFS) measurement error. Note that the increased amplitude measured for the probe wave for longer code lengths distorts the measured spectra that, compared to that measured with no coding, is narrowed and skewed towards the BFS of the fiber outside the hotspot. However, as shown in Fig. 4 (b), our response linearization method completely compensates this effect and the spectra for the pulsecoded BOTDA are seen to coincide with those of the single-pulse sensor, just with less noise due to the coding gain.

Figure 5 depicts the improvement on the BFS measured along the sensing fiber by applying our response linearization technique for a code length $L_{c}=251$. Notice that this link is made of two fiber spools with slightly different average BFS. The insets on Fig. 5 show a zoom of the measurement at both hotspots. It can be seen that for the system without linearization there is the previously mentioned approx. 2-MHz error in the measured BFS at the first hotspot for increasing code length, which is due to the spectral biasing depicted in Fig. 4 (a). This measurement error increases strongly after $54 \mathrm{~km}$, however, this error is successfully corrected altogether by deploying the linearization method. For the second hotspot just the BFS linearized result is shown because without linearization the Brillouin spectrum at that location was completely distorted.

Figure 5 includes an additional measurement of the sensing 

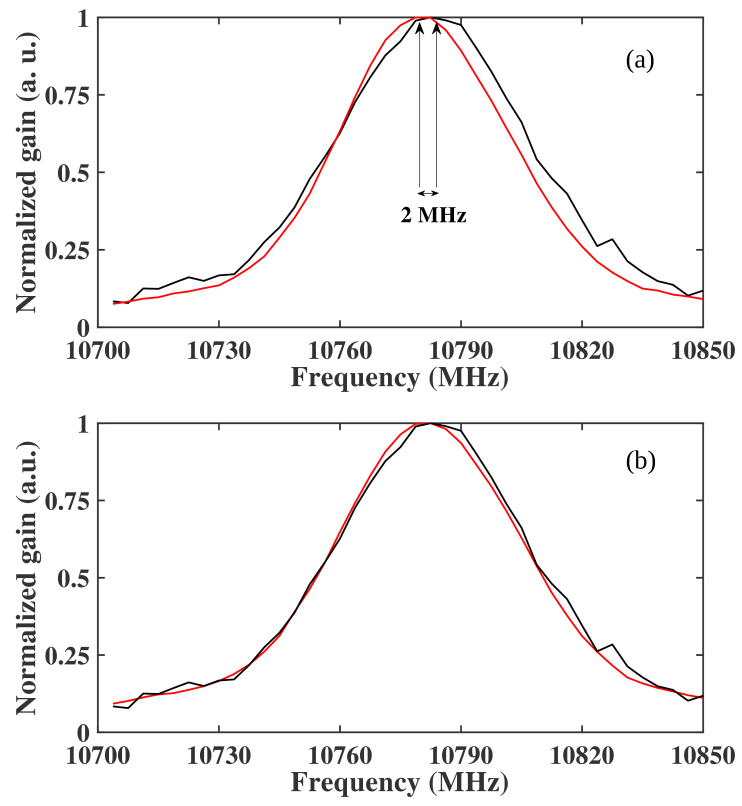

Fig. 4. Brillouin spectra measured at the hotspot near the start of the fiber (a) without and (b) with linearization of the sensor response for code lengths of $L_{c}=1$ (no coding) (black) and $L_{c}=251$ (red).

fiber swapping the two fiber ends. This measurement highlights that from around $80 \mathrm{~km}$ of sensing fiber there is a deviation of the BFS from its true value with oscillation and biasing introduced. However, this error was neither due to nonlocal effects, nor to nonlinear effects such as self-phase modulation or modulation instability affecting the pulses. This was confirmed by repeating the measurements after reducing either the pump or the probe wave power and simultaneously increasing the number of averages to maintain the SNR of the detected signal. Identical BFS distributions were obtained in all cases independently of the pump and probe power. We finally attributed the small observed variation of BFS to residual errors in the amplitude weighting of the pump pulses sequences introduced in the decoding process to account for the variation of their relative power at the output of the EDFA. We found that the decoded measurements and the obtained BFS distribution were very sensitive to small variations in those weights, leading to the sort oscillations that we were observing. Nevertheless, the objective of this work was to introduce a proof-of-concept experiment demonstrating the linearization method. The full potential of the coding linearization technique in terms of measurement range will be explored with further work, probably deploying an additional method to obtain flat amplitude pulses at the output of the EDFA [2, 7].

Finally, Fig. 6 depicts the uncertainty of BFS measurements along the fiber calculated as the standard deviation of 8 consecutive measurements. A remarkable performance is obtained assuming an $80-\mathrm{km}$ sensing link (within a total $200-\mathrm{km}$ fiber loop) with a measurement precision better than $2 \mathrm{MHz}$ for 16000 trace averages and 2-m spatial resolution, obtaining a figure-ofmerit of 4400, while if linearization method is not applied the figure of merit is 84 .

In summary, we have introduced a very simple method to extend the applicability of pulse coding in BOTDA sensors by compensating the effects of the nonlinear amplification of the probe wave by a sequence of pulses. Notice that during the

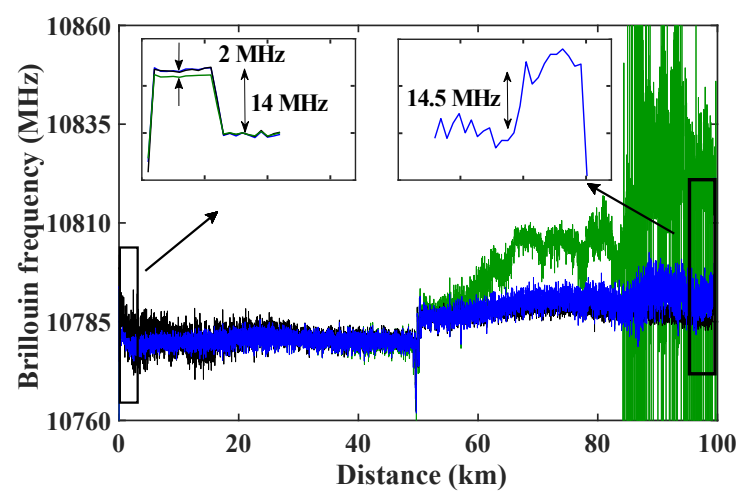

Fig. 5. BFS measured along the fiber for $L_{c}=251$ with coding linearization (blue and black) and without linearization (green): blue and black traces correspond to measurements swapping the two fiber ends. Insets: BFS measured at the two hotspots for: $L_{c}=1$ (no coding) (black), $L_{c}=251$ without linearization (green) and $L_{c}=251$ with linearization (blue).

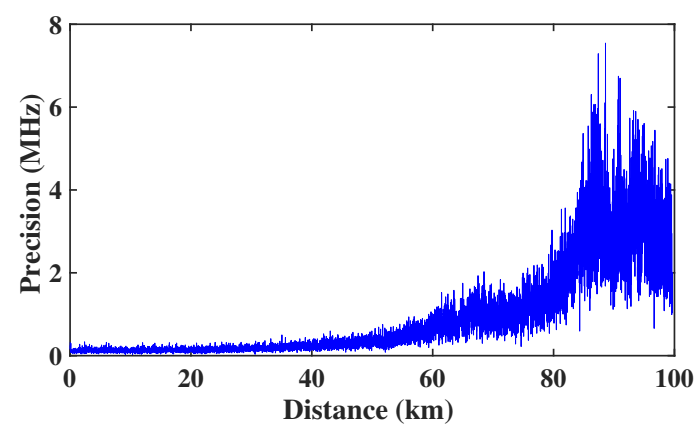

Fig. 6. BFS measurement precision along the fiber (standard deviation of 8 consecutive measurements).

submission and revision process of this work, a similar coding linearization method has been published [8]. The contribution of our work, apart from the independently-developed demonstration of the technique, is its application to a much longer $200-\mathrm{km}$ link. Furthermore, this new work combines linearization with the probe dithering technique to get rid of the nonlocal effects that are exacerbated by coding.

Funding. TEC2016-76021-C2-1-R, (AEI/FEDER,UE), UPNA.

\section{REFERENCES}

1. H. Liang, W. Li, N. Linze, L. Chen, and X. Bao, Opt. Lett. 35, 1503 (2010).

2. S. L. Floch, F. Sauser, M. Llera, and E. Rochat, J. Light. Technol. 33, 2623 (2015).

3. M. A. Soto, S. L. Floch, and L. Thévenaz, Opt. Express 21, 16390 (2013).

4. H. Iribas, A. Loayssa, F. Sauser, M. Llera, and S. L. Floch, Opt. Express 25, 8787 (2017).

5. R. Ruiz-Lombera, J. Urricelqui, M. Sagues, J. Mirapeix, J. M. LópezHiguera, and A. Loayssa, IEEE Photonics J. 7, 1 (2015).

6. M. Taki, Y. Muanenda, C. J. Oton, T. Nannipieri, A. Signorini, and F. D. Pasquale, Opt. Lett. 38, 2877 (2013).

7. F. Wang, C. Zhu, C. Cao, and X. Zhang, Opt. Express 25, 3504 (2017).

8. Z. Yang, Z. Li, S. Zaslawski, L. Thévenaz, and M. A. Soto, Opt. Express 26, 16505 (2018). 